\title{
Survey on Phenomenon Mobility Prediction using Virtual Force-based Interest Driven Monitoring Scheme
}

\author{
Rakhi Narkhede ${ }^{1}$, Prof. L. H. Patil ${ }^{2}$, Prof. U. K. Thakur ${ }^{3}$ \\ ${ }^{1}$ Research Scholar, Department of Computer Science and Engineering, Priyadershini Institute of Engineering and Technology Nagpur \\ ${ }^{2}$ Professor, Department of Computer Science and Engineering, Priyadershini Institute of Engineering and Technology \\ ${ }^{3}$ Professor, Department of Computer Science and Engineering, Priyadershini Institute of Engineering and technology, RTMNU, Nagpur
}

\begin{abstract}
In recent years, the technology is being growing very fast. Many new techniques have been introduced in Mobile Sensor Network. A new technique that is a monitoring scheme is developed for monitoring the moving phenomenon using virtual force called virtual force-based interest driven monitoring scheme. The scheme is evaluated in three variants: VirFID-LIB, VirFID-GHL and VirFID-IBN. All these three algorithms perform different action on the MSN to achieve the highest interest value. It is possible to extend this scheme for predicting the next movement of the phenomenon. It can be done by creating the database of few early moves of the phenomenon and then apply the prediction algorithm to the appropriate data. This will result in the prediction about the next possible move of the phenomenon.
\end{abstract}

Keywords: Monitoring scheme, interest value, virtual force, database, prediction

\section{Introduction}

A mobile sensor network, multiple mobile sensor nodes cooperate with other to achieve a common goal. In VirFID, there is a of multiple autonomous mobile sensor nodes. MS nodes are equipped with sensing devices to collect data on the phenomenon. MS nodes are also equipped with communication devices to communicate with other MS nodes or to send data to root node. A phenomenon is a set of points where a meaningful sensing value can be obtained. Meaningful sensing value depends on the application and the monitoring target. Phenomenon is regarded as moving, if the target of interest is relocated due to some external environmental factor.

The weighted sensing coverage denotes the weighted sum of covered areas. The interest value is used as the weight of the area. A high weighted sensing coverage is achieved when an MSN can cover the region of highest interest value as wide as possible. When the MSN is monitoring the phenomenon, nodes placement need to be optimized such that the maximum weight is monitoring the phenomenon, nodes placement need to be optimized such that the maximum weighted sensing coverage can be obtained. The weighted sensing coverage is defined as the weighted sum of interest value multiplied by the size of unit area in coverage.

Weighted coverage efficiency is a term which shows how effectively nodes can cover the area of interest. It is defined as the ratio of the weighted sensing coverage obtained by the nodes to the maximum weighted sensing coverage that can be obtained in the entire area of interest. Simply, the weighted coverage efficiency denotes that how close the current WSC is to the maximum WSC and also that how close is the distribution of the nodes to the optimal.

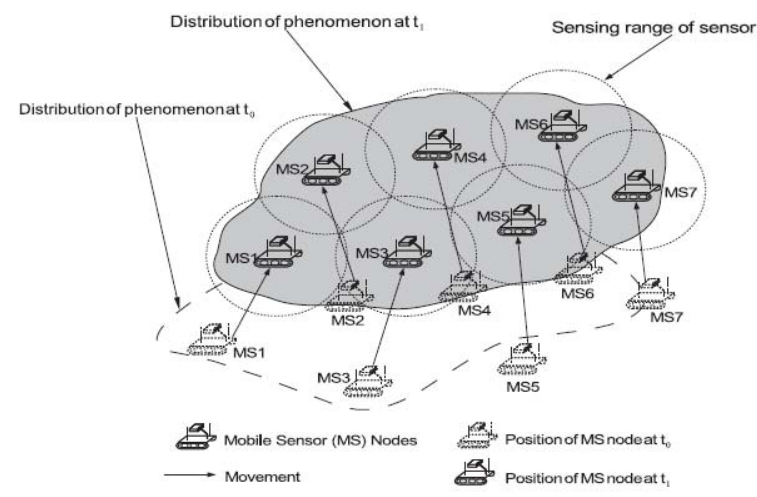

Figure 1: An example of moving phenomenon monitoring using mobile sensor nodes

A database is created which contain all the data about the previous movements of the phenomenon which is being monitored by the mobile sensor nodes. A data mining algorithm is applying to this previous data which gives the large mobility pattern of the phenomenon. After obtaining the large pattern, mobility rules are set. Which means a set of mobility rules is created. This set of rules is then applied to the obtained large patterns which results in the prediction of the phenomenon.

\section{Literature Survey}

Dynamic Sensor Deployment Algorithm: Virtual force is an approach for dynamic sensor deployment. Virtual force concept comes from the potential field technique. In this algorithm mobile sensor nodes are initially placed at random positions. After initial deployment, the sensor nodes are moved according to the distance between sensors [1].

The mobile user moves in a wireless network which has GSM standard. The coverage area of the network is 


\section{International Journal of Science and Research (IJSR) \\ ISSN (Online): 2319-7064}

Index Copernicus Value (2013): 6.14 | Impact Factor (2014): 5.611

partitioned into the smaller area called cells. The coverage areas consist of the number of location area. Each location area consist of one or more cells [2]. ID of the cells are regularly broadcasted by the base station. Sequential pattern mining is applied to the domain of predictive Web prefetching. User access patterns are mined from the web logs of user's previous requests which is helpful for the user's future prediction.

First phase of the algorithm gives the user mined pattern, second phase gives the candidates in the pattern and the last phase gives the prediction about the user.

Locational optimization problems pervade a broad spectrum of scientific disciplines. Biologists rely on locational optimization tools to study how animals share territory and to characterize the behavior of animal groups obeying the following interaction rule: each animal establishes a region of dominance and moves toward its center.[3]

Proposes a virtual force directed evolutionary particle swarm optimization (VFCPSO) algorithm, which uses a combined objective function to achieve the tradeoff of coverage and energy consumption. By considering deployment as an optimization problem, VFCPSO is more reliable and flexible for WSNs, since it can satisfy the combined requirements instead of only enlarging coverage. It is more efficient than other three VFCPSO algorithms and the VF-style algorithms in terms of computation time, coverage and efficient moving energy consumption [4].

Present a novel approach to localization of sensors in a network given a subset of noisy intersensor distances. The algorithm is based on "stitching" together local structures by solving an optimization problem requiring the structures to fit together in an "As-Rigid-As-Possible" manner, hence the name ARAP. The local structures consist of reference "patches" and reference triangles, both obtained from intersensor distances. We elaborate on the relationship between the ARAP algorithm and other state-of-the-art algorithms, and provide experimental results demonstrating that ARAP is significantly less sensitive to sparse connectivity and measurement noise [5].

In WSANs, sensors gather information about the physical world, while actors take decisions and then perform appropriate actions upon the environment, which allows a user to effectively sense and act from a distance. In order to provide effective sensing and acting, coordination mechanisms are required among sensors and actors. Moreover, to perform right and timely actions, sensor data must be valid at the time of acting [7]

The proposed methods are implemented on a Web log to estimate their effectiveness. It is shown through performance experiments that the proposed rule-based methods are effective in improving the system performance in terms of the average latency as well as the cache hit ratio of mobile clients [8].

Several algorithms have been proposed, which find important applications, like user profiling, recommender systems, web prefetching, design of adaptive web sites, etc.
In all these applications the core issue is the developement of an effective prediction algorithm. In [9], focus is on webprefetching, because of its importance in reducing user perceived latency present in every Web-based application.

[10] method has performed well with a variety of corruption factor and outlier percentage values. We have observed that although an increase in the corruption in the data decreases the recall and precision, an increase in the outlier percentage has no significant effect on the recall and precision.

When compared to the performance of the baseline method, which is Ignorant Prediction, our method provides a very good performance in terms of precision and recall.

[11] present a theoretical framework for design and analysis of distributed flocking algorithms. Two cases of flocking in free-space and presence of multiple obstacles are considered. We present three flocking algorithms: two for free-flocking and one for constrained flocking. A comprehensive analysis of the first two algorithms is provided. We demonstrate the first algorithm embodies all three rules of Reynolds. This is a formal approach to extraction of interaction rules that lead to the emergence of collective behavior.

\section{Proposed System}

VirFID algorithms are designed for monitoring the moving phenomenon, in which the movements of nodes are controlled to form a sensor network and follow the phenomenon for long-term monitoring. The scheme is evaluated in three variants, VirFID-LIB(Local Information Based), VirFID-GHL( Global Highest Lowest) and VirFIDIBN(Interest at Boundary Nodes). Local Information Based algorithm uses only local neighborhood information sharing among nodes. As the nodes in VirFID-LIB uses only local information, the nodes may suffer from the local maxima problem. Also, they cannot adjust their position to quickly explore uncovered regions of higher interest values.

VirFID-Global Highest Lowest shares the highest and lowest interest values and the position of the nodes that has those values. VirFID-GHL uses spatial locality in phenomenon distribution. In this a higher interest value may be found near positions where the highest value is already obtained. In this algorithm, first it determines the highest and lowest interest value. Depending on these values, each boundary node determines its relative extent in terms of interest value. Thus the nodes in MSN adjust their position to achieve the highest interest value, which may lead in expansion of the network or even contraction of the network.

VirFID-Interest at Boundary Nodes distributes the nodes in such a way that is close to the optimum. The boundary nodes are likely to have similar interest values. VirFID-IBN attempts to assign the same interest value to all boundary nodes. Firstly, it collects the interest value of boundary nodes, and then mean interest value is calculated and published to all boundary nodes which help them to calculate the force need to apply using the mean interest value as shown in fig 2 . 


\section{International Journal of Science and Research (IJSR) \\ ISSN (Online): 2319-7064}

Index Copernicus Value (2013): 6.14 | Impact Factor (2014): 5.611

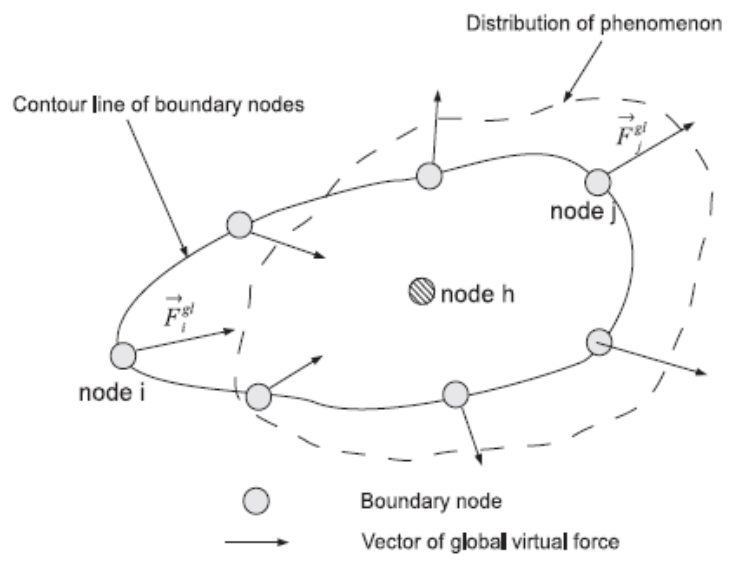

Figure 2: An example of operation of VirFID-IBN algorithm.

All the movements of the phenomenon are recorded by the sensor nodes which are following the particular phenomenon. Database is created where all this data is saved in an appropriate manner. Whenever needed the data is extracted from the database about the phenomenon which is then used as an input by the user mobility pattern algorithm.

Mobility prediction is one of the most essential issues that need to be explored for mobility management in mobile computing system. A new algorithm for prediction is proposed which consist of three phases. In first phase, the user mobility patterns are mined from the history. In second phase, mobility rules are extracted from the patterns and in the third phase, mobility predictions are accomplished by using these rules.

A new method is designed that is convenient by generalizing the method and applying it for user mobility pattern mining. In UMP algorithm, a large pattern is generated by appending the candidates to each other. Candidates having enough support value are only added to the pattern. Input to this algorithm is the data from the database that is the actual path of the phenomenon. After the large patterns are generated, candidates of those patterns are generated and then the mobility rules.

Set of mobility rules play a very important role in the prediction algorithm. In the third phase that is the predictions phase, these mobility rules are applied to patterns. According to the rules and the confidence value of the candidates, the algorithm returns the prediction about the phenomenon.

\section{Conclusion}

In VirFID, to maximize the weighted sensing coverage mobile sensor nodes repeated place themselves at the positions where higher interest sensing data is obtained by applying the virtual force which is based on the distance between the mobile sensor node and the sensed value in the area of interest. MS nodes perform sharing of information about their interest value and their positions to achieve the highest weighted sensing coverage. VirFID can be extended such that it can predict the behavior of the phenomenon based on its previous movements. Here, a data mining algorithm can be used to mine the appropriate data about the particular phenomenon from the database. A mobility pattern can be obtained from the actual path of the phenomenon. To this pattern then the mobility rules are applied, which result in the prediction about that phenomenon.

\section{References}

[1] Duc Van Le, Hoon Oh, Seokhoon Yoon," VirFID: A Virtual Force (VF)-based Interest-Driven moving phenomenon monitoring scheme using multiple mobile sensor nodes", Ad Hoc Networks 27 (2015).

[2] GokhanYavas, DimitriosKatsaros, OzgurUlusoy, YannisMano;opoulos, "A data mining approach for location prediction in mobile environments", data and knowledge engineering 54 (121-146)

[3] J. Cortes, S. Martinez, T. Karatas, F. Bullo "Coverage control for mobile sensing networks" IEEE Trans. Robot. Automation, 20 ,pp. 243-255

[4] X. Wang, S. Wang, Hierarchical deployment optimization for wireless sensor networks, IEEE Trans. Mobile Comput. 10 (2011) 1028-1041.

[5] C.G.L. Zhang, L. Liu, S.J. Gortler, "An as-rigid-aspossible approach to sensor network localization", ACM Trans. Sensor Netw. 6 (2010) 35:1-35:21.

[6] S. Rajagopal, R.B. Srinivasan, R.B. Narayan, X.B.C. Petit, "GPS-based predictive resource allocation in cellura networks", in: Proceedings of the IEEE International Conference on Networks (IEEE ICON_02), pp. 229-234

[7] I.F. Akyildiz, I.H. Kasimoglu "Wireless sensor and actor networks: research challenges" Ad Hoc Network., 2 , pp. 351-367

[8] Y. Saygin, O. Ulusoy, "Exploiting data mining techniques for broadcasting data in mobile computing environments", IEEE Trans. Knowl. Data Eng. 14 (6) 1387-1399

[9] A. Nanopoulos, D. Katsaros, Y. Manolopoulos," Effective prediction of web user accesses: a data mining approach", in: Proceedings of the WebKDD Workshop

[10] A. Aljadhai, T. Znati, "Predictive mobility support for QoS provisioning in mobile wireless environments", IEEE J. Select. Area Commun

[11]Reza Olfati-Saber," Flocking for Multi-Agent Dynamic Systems", IEEE transactions on automatic control, vol. 51, no. 3 